\title{
Cold exposure impairs dark-pulse capacity to induce REM sleep in the albino rat
}

\author{
FRANCESCA BARACCHI ${ }^{1,2}$, GIOVANNI ZAMBONI ${ }^{1}$, MATTEOCER RI ${ }^{1}$, \\ ELIDE DEL SINDACO ${ }^{1}$, DANIELA DENTICO ${ }^{1}$, CHRISTINE ANN JONES ${ }^{1}$, \\ MARCO LUPPI ${ }^{1}$, EMANUELE PEREZ ${ }^{1}$ and ROBERTO AMICI ${ }^{1}$ \\ ${ }^{1}$ Department of Human and General Physiology, Alma Mater Studiorum-University of Bologna, Italy and ${ }^{2}$ Department of Anesthesiology, \\ Research Division, University of Michigan, Ann Arbor, USA
}

Accepted in revised form 24 February 2008; received 30 November 2007

SUMMARY In the albino rat, a REM sleep (REMS) onset can be induced with a high probability and a short latency when the light is suddenly turned off (dark pulse, DP) during nonREM sleep (NREMS). The aim of this study was to investigate to what extent DP delivery could overcome the integrative thermoregulatory mechanisms that depress REMS occurrence during exposure to low ambient temperature (Ta). To this aim, the efficiency of a non-rhythmical repetitive DP (3 min each) delivery during the first 6-h light period of a $12 \mathrm{~h}: 12 \mathrm{~h}$ light-dark cycle in inducing REMS was studied in the rat, through the analysis of electroencephalogram, electrocardiogram, hypothalamic temperature and motor activity at different Tas. The results showed that DP delivery triggers a transition from NREMS to REMS comparable to that which occurs spontaneously. However, the efficiency of DP delivery in inducing REMS was reduced during cold exposure to an extent comparable with that observed in spontaneous REMS occurrence. Such impairment was associated with low Delta activity and high sympathetic tone when DPs were delivered. Repetitive DP administration increased REMS amount during the delivery period and a subsequent negative REMS rebound was observed. In conclusion, DP delivery did not overcome the integrative thermoregulatory mechanisms that depress REMS in the cold. These results underline the crucial physiological meaning of the mutual exclusion of thermoregulatory activation and REMS occurrence, and support the hypothesis that the suspension of the central control of body temperature is a prerequisite for REMS occurrence.

KEYWORDS dark pulse, low ambient temperature, non-REM sleep to REM sleep transition, preotpic-anterior hypothalamus, REM sleep, REM sleep homeostasis

\section{INTRODUCTION}

The wake-sleep cycle consists of the alternation of three different states, Wake, non-REM sleep (NREMS) and REM sleep (REMS), which are usually identified on the basis of the level of brain cortical and muscle activity. From a physiological point of view, the major feature that differentiates REMS from both Wake and NREMS consists of an operational

Correspondence: Roberto Amici, Department of Human and General Physiology, Alma Mater Studiorum-University of Bologna, Piazza P.ta S. Donato, 2 I-40126 Bologna, Italy. Tel.: + 39-051-2091735; fax: +39-051-2091737; e-mail: roberto.amici@unibo.it change in physiological regulation that shifts from a homeostatic to a non-homeostatic modality during REMS (Parmeggiani, 2005). Such a shift is more relevant for a regulation, such as thermoregulation, that needs a high degree of integration and largely depends on the activity of regulatory structures of the hypothalamus (Parmeggiani, 2003). Thus, REMS occurrence needs to be finely regulated, since it represents a physiological challenge for the organism, particularly when under unfavourable ambient conditions, such as during the exposure to a low ambient temperature (Ta).

In different species, the passage from NREMS to REMS has been shown not to occur abruptly (Gottesmann, 1996; 
McCarley and Hobson, 1970). In the rat, REMS episodes are always preceded by a short period (30-60 s), which has been defined 'NREMS to REMS transition' and is characterized by a specific pattern of the electroencephalogram (EEG), where a fall in Delta activity is paralleled by an increase in both Theta and Sigma activity (Benington et al., 1994; Capitani et al., 2005; Trachsel et al., 1988). However, in some cases, the transition does not lead to a REMS onset and is followed by an awakening. The probability for a transition to be successfully followed by a REMS onset has been shown to be quantitatively related to the presence of specific EEG and autonomic patterns during its occurrence (Benington et al., 1994; Capitani et al., 2005; Parmeggiani et al., 1975). In particular, in both the cat and the rat the pattern of changes in hypothalamic temperature (Thy) appears to be critical (Capitani et al., 2005; Parmeggiani et al., 1975). On this basis, the NREMS to REMS transition may be considered as a dynamic process during which the physiological challenge of entering REMS is weighted at the level of the regulatory structures of the hypothalamus.

In spite of this apparently tight control, in 1966 Lisk and Sawyer showed that a REMS onset could be induced with a high probability and a short latency in the albino rat when the light was suddenly turned off (dark pulse, DP) during NREMS. This REMS-inducing effect of DP was not observed in non-albino rats (Benca et al., 1990) and only a mild short latency REMS enhancing the effect of light-off was recently observed in C57BL/6 mice exposed to short light-dark cycles (Deboer et al., 2007). It has therefore been hypothesized that the effect is due to components of the visual system affected by albinism (Lund, 1965), which are part of a circuitry acting on the brain-stem nuclei involved in REMS expression (Fleming et al., 2006; Miller et al., 1996). Among them, the role of pretectal area (PT) would appear to be crucial (Miller et al., 1998, 1999). Even so, the enhancement of REMS occurrence due to a rhythmical DP delivery does not increase the daily amount of REMS, due to an apparent compensatory decrease in REMS occurrence during the periods between DPs in which the light is on. On this basis, it has been suggested that the DP effect may be mediated by the same mechanisms that regulate spontaneous REMS onset (Rechtschaffen et al., 1969).

Up to now, the efficacy of DP has not been tested under conditions that are known to challenge REMS occurrence. In particular, no studies have been carried out in which DP has been delivered to animals kept at low Ta, which represents a physiological challenge known to interfere with REMS expression. The exposure to low ambient temperature is known to depress REMS occurrence in different species to an extent that is related to the degree of Ta lowering (Cerri et al., 2005; Heller, 2005; Parmeggiani, 2003). Such a depression can be interpreted as a specific thermoregulatory response favouring the occurrence of behavioural states in which autonomic thermoregulation is fully operant (Zamboni et al., 2004). On this basis, it appears to be of interest to test whether DP (which is the most efficient REMS-inducing stimulus in the albino rat, thought to function by directly acting on the brain- stem nuclei involved in REMS expression) is able to overcome the tonic depressing influence of central thermoregulatory activation on REMS occurrence.

To this aim, the effects of DP delivery were studied in animals kept at either normal laboratory ambient temperature $\left(\mathrm{Ta}, 25^{\circ} \mathrm{C}\right)$ or low $\mathrm{Ta}\left(15^{\circ} \mathrm{C}\right)$ during the first $6 \mathrm{~h}$ of the light period of a $12 \mathrm{~h}: 12 \mathrm{~h}$ light-dark (LD) cycle, when nonrhythmical and automatic DPs were delivered independently from the wake-sleep state. The post-effects during the following $18 \mathrm{~h}$-period were also analysed. Also, the time course of both the electroencephalogram (EEG) and an autonomic variable, i.e. Thy, were analysed during either spontaneous or DP-induced 'NREMS to REMS transitions'. The results of this experiment showed that in animals exposed to low Ta, DP could not overcome the integrative thermoregulatory mechanisms which depress spontaneous REMS occurrence, since its efficacy was depressed to an extent that was comparable with that observed in spontaneous REMS occurrence.

However, at low Ta, DPs were often delivered during NREMS episodes which were not fully consolidated, characterized by an average Delta power that was significantly lower than that observed at Ta $25^{\circ} \mathrm{C}$. Thus, a second experiment was carried out in which DPs were delivered manually by the experimenter. This procedure allowed more strict criteria for DP delivery, i.e. only during consolidated NREMS episodes (>2 min) with a stable Delta activity. Also, it allowed us to avoid any possible interference with the efficiency of DP delivery during NREMS caused by DP delivery during either Wake or REMS. Moreover, a further physiological variable, i.e. electrocardiogram (EKG), was studied. The results of this experiment substantially confirmed those of the first one, leading to the conclusion that the efficacy of DPs at low Ta is dampened by the overall neurophysiologic and autonomic state of the animal resulting from thermoregulatory activation. Preliminary results of the present study have been published in abstract form (Baracchi et al., 2004).

\section{METHODS}

Twenty-two male Sprague-Dawley rats (Charles River), which had free access to food and water and were kept at $25 \pm 0.5^{\circ} \mathrm{C} \mathrm{Ta}$, under a $12 \mathrm{~h}: 12 \mathrm{~h}$ LD cycle (L: 09:00 21:00 h; 100 lux at cage level), were used. The experiments were carried out according to the European Union Directive (86/609/EEC) and were under the supervision of the Central Veterinary Service of the University of Bologna and the National Health Authority.

\section{Surgery}

Animals were placed under deep general anaesthesia (diazepam, Valium, Roche, $5 \mathrm{mg} \mathrm{kg}^{-1}$ i.m.; ketamine-HCl, Ketalar, Parke-Davis, $100 \mathrm{mg} \mathrm{kg}^{-1}$ i.p.) and were implanted epidurally on the right-hand side with two stainless steel electrodes for frontal-parietal electroencephalographic (EEG) recording 
(3.0 $\mathrm{mm}$ anterior, $3.0 \mathrm{~mm}$ lateral to Bregma; $4.0 \mathrm{~mm}$ posterior, $1.5 \mathrm{~mm}$ lateral to Bregma).

A thermistor (B10KA303N, Thermometrics) mounted inside the tip of a stainless steel needle ( 21 gauge) was positioned above the left anterior hypothalamus to measure hypothalamic temperature (Thy). The plastic plugs used to connect the EEG electrodes and the thermistor to the recording apparatus were embedded in acrylic dental resin anchored to the skull by small stainless steel screws, which had also been implanted epidurally at the outer limit of the surgical field.

EKG was recorded in selected animals (see experiment II). Two stainless steels wires insulated with polyethylene were used as electrodes (Cooner Wire). The longer one $(6.0 \mathrm{~cm})$ was fixed at the posterior part of the xiphoid process, while the shorter one $(4.0 \mathrm{~cm})$ was fixed at the dorsal part of the sternomastoideus muscle and its extremity $(0.5 \mathrm{~cm})$ was free to float within the mediastinum. During the experimental sessions, the behaviour of the animals was followed by means of a closed-circuit video system.

\section{Experimental plan}

During each experimental session, recording started at 09:15 h, after $15 \mathrm{~min}$ spent on animal care, and ended at 09:00 $\mathrm{h}$ of the subsequent day. Two experiments were carried out.

Experiment I: automatic dark-pulse delivery at either normal laboratory or low ambient temperature

Fourteen animals were used. The experimental plan consisted of four different experimental 24-h sessions:

(i) Normal laboratory $\mathrm{Ta}[\mathrm{N}]$ : animals were kept at Ta $25^{\circ} \mathrm{C}$;

(ii) Dark-pulse delivery at normal laboratory $\mathrm{Ta}$ [NDP]: animals were kept at Ta $25{ }^{\circ} \mathrm{C}$. Dark pulses were delivered during the first $6 \mathrm{~h}$ of the L period (09:00-15:00 h);

(iii) Exposure to low $\mathrm{Ta}[\mathrm{E}]$ : animals were exposed to $\mathrm{Ta}$ $15^{\circ} \mathrm{C}$ during the first $6 \mathrm{~h}$ of the $\mathrm{L}$ period (09:00-15:00 h) and then were returned to Ta $25^{\circ} \mathrm{C}$;

(iv) Dark-pulse delivery during the exposure at low Ta [EDP]: animals were exposed to Ta $15^{\circ} \mathrm{C}$ during the first $6 \mathrm{~h}$ of the $\mathrm{L}$ period (09:00-15:00 $\mathrm{h}$ ) and then were returned to Ta $25{ }^{\circ} \mathrm{C}$. Dark pulses were delivered during the 6-h exposure to low Ta.

In order to balance any possible carry-over effect of the first DP-delivery session on the second one, two experimental protocols were used, A $(n=7)$ and $\mathrm{B}(n=7)$. Under protocol A, the sequence of the experimental sessions was (i), (ii), (iii) and (iv). Both sessions (i) and (ii) were carried out on 2 consecutive days, followed by 2 days without experiments and, finally, sessions (iii) and (iv) were carried out on last 2 consecutive days. Under protocol B, the schedule was reversed and sessions (iii) and (iv) were followed after 2 days without experimental sessions (i) and (ii).

Dark-pulse stimulation consisted of 20 stimuli each lasting $3 \mathrm{~min}$, delivered automatically by means of a software-driven remote control of the cage lighting. Stimuli were delivered non- rhythmically, at fixed hours (minimum interval between two consecutive DP: $10 \mathrm{~min}$; frequency: 4/h), independently from the stage of the wake-sleep cycle. In order not to interfere with the start of a normal wake-sleep cycle, the first stimulus was delivered not before 10:00 h. DP was considered to be effective in inducing a REMS onset if it was followed by a REMS onset within the duration of the DP (3 min).

Experiment II: manual dark-pulse delivery at either normal laboratory or low ambient temperature

Eight animals were used. As for experiment I, animals were implanted with EEG electrodes and a thermistor, but two electrodes for EKG recording were added. Due to the presence of artefacts in two animals, EKG data from only six animals will be shown. The experimental plan consisted of two different 6-h experimental sessions (09:00-15:00 h), which were carried out on 2 consecutive days. This experiment substantially aimed to repeat treatments (ii) and (iv) of experiment I:

(i) Dark-pulse delivery at normal laboratory Ta: dark pulses were delivered during the first $6 \mathrm{~h}$ of the L period (09:0015:00 h) to animals kept at Ta $25^{\circ} \mathrm{C}$.

(ii) Dark-pulse delivery at low Ta: dark pulses were delivered during the first $6 \mathrm{~h}$ of the $\mathrm{L}$ period $(09: 00-15: 00 \mathrm{~h})$ to animals acutely exposed to Ta $15^{\circ} \mathrm{C}$.

Frequency analysis of the EEG was carried out online and animal behaviour was monitored by means of a closed-circuit video control. Differently from what happened in experiment I, DPs were delivered by the experimenter, who switched off the light manually. Ten DPs, lasting 3 min each, were delivered in each session according to the following criteria:

(i) Presence of a consolidated NREMS episode ( $>2 \mathrm{~min}$ ) with a stable Delta activity $(0.75-4.0 \mathrm{~Hz})$.

(ii) Presence of a low Sigma activity $(11.0-16.0 \mathrm{~Hz})$, in order to avoid delivery during a NREMS to REMS transition (Benington et al., 1994; Capitani et al., 2005; Trachsel et al., 1988).

(iii) Not less than $12 \mathrm{~min}$ from the end of the previous DP.

(iv) Not less than $5 \mathrm{~min}$ from the end of the last REMS episode.

(v) No more than 3 stimuli per hour.

Due to the strictness of these criteria, only nine DPs were delivered to one animal at Ta $25{ }^{\circ} \mathrm{C}$ and seven animals at Ta $15{ }^{\circ} \mathrm{C}$.

\section{Signal analysis}

User software was developed (QuickBASIC) in order to handle the data. In each experiment, the EEG signal was amplified, filtered (high-pass filter: $-40 \mathrm{~dB}$ at $0.35 \mathrm{~Hz}$; low-pass filter: $-6 \mathrm{~dB}$ at $60 \mathrm{~Hz}$; digital Notch filter: $-40 \mathrm{~dB}$ at $50 \mathrm{~Hz}$ ) and AD converted (sampling rate: EEG, $128 \mathrm{~Hz}$ ). The EEG signal was subjected to online Fast Fourier Transform (FFT) and EEG power values were obtained for 4-s epochs in the Delta $(0.75$ 4.0 Hz), Theta $(5.5-9.0 \mathrm{~Hz})$ and Sigma $(11.0-16.0 \mathrm{~Hz})$ band. Thy signal was amplified $\left(1^{\circ} \mathrm{C} / 1 \mathrm{~V}\right)$ before $\mathrm{AD}$ conversion 
(sampling rate: $8 \mathrm{~Hz}$ ). Motor activity (MA) was monitored by means of a passive infrared detector (Siemens, PID20) placed at the top of each cage. The signal was amplified and integrated before $\mathrm{AD}$ conversion (sampling rate: $8 \mathrm{~Hz}$ ), in order to make the output proportional to the amplitude and the duration of movement. This system detected most of the movements related to the normal behaviour of the rat, such as exploring, grooming, feeding and small movements such as twitching or brief awakenings in either REMS or NREMS. The EKG signal was amplified, filtered (high pass: $3 \mathrm{~Hz}$; low pass: $100 \mathrm{~Hz}$ ) and $\mathrm{AD}$ converted (sampling rate: EKG, $1024 \mathrm{~Hz}$ ). Following AD conversion, all signals were stored on a $\mathrm{PC}(486 / 100 \mathrm{DX}-4)$.

\section{Data analysis and wake-sleep staging}

EEG, Thy and MA signals were visually scored in order to assess REMS parameters, i.e. number and duration of REMS episodes and total amount of REMS. The main criteria used for this assessment were based on the analysis of EEG (increase in theta power and decrease in Delta and Sigma power) and MA (absence of movements) (Amici et al., 1994, 1998; Cerri et al., 2005). Furthermore, particular consideration was given to changes in hypothalamic temperature, which is considered to be an index of state-dependent changes in autonomic activity and is known to increase at REMS onset and to decrease at REMS end (Parmeggiani, 2007). According to this, a REMS episode was considered as such only if the EEG and MA changes associated with this sleep stage were concomitant with an increase in Thy. Moreover, the end of a REMS episode was considered as such only if the EEG and MA changes which characterize an awakening were associated with a decrease in Thy. The duration for the minimal duration of either a REMS episode or a brief awakening was fixed at $8 \mathrm{~s}$. Following visual scoring for REMS and the removal of the $4 \mathrm{~s}$ epochs showing EEG artefacts (which were less than $1 \%$ and were visually scored at the end of the procedure), Wake and NREMS were automatically separated by using a data transformation previously described (Cerri et al., 2005), which takes into account the levels of delta and Sigma power and MA in the different 4-s epochs. The match between automatic and visual scoring has been shown to be well above 95\% (Cerri et al., 2005).

\section{Statistical analysis}

Statistical analysis was carried out by means of SPSS 9.0. The analysis of the time spent in Wake, NREMS and REMS was carried out using two-way repeated measure ANOvA, according to an $a \times b$ factorial plan where factor a represented Ta during the first $6 \mathrm{~h}$ of the experimental session (factor Ta, two levels: $25^{\circ} \mathrm{C}, 15^{\circ} \mathrm{C}$ ) and factor $b$ represented dark-pulse delivery (factor DP, two levels: DP-, DP + ). Separate analyses were carried out for the first 6-h block (09:00-15:00 h), the second 18-h block (15:00-09:00 h) and the whole 24-h period (09:00 09:00 h).
The analysis of delta power density, a variable which is known to be finely modulated hour by hour across the 24-h period, was carried out using three-way repeated measures ANOVA in which factor $c$, which represented time of day (factor time, 12 levels, consisting of consecutive 2-h blocks), was also considered in addition to factors Ta and DP.

The analysis of the time course of the power densities in the different bands of EEG, Thy levels and heart rate before and during dark-pulse delivery was carried out using one-way repeated measures ANOvA.

Post hoc individual comparisons were carried out by means of the modified $t$-test $\left(t^{*}\right)$ and Bonferroni's correction for multiple comparisons (Wallenstein et al., 1980).

Analysis of the latency (median value) of the response to the DP delivery and that of the efficacy of DP delivery in the different experimental conditions was carried out by means of the Friedman's test and the chi-square test, respectively.

\section{RESULTS}

\section{Experiment 1}

Analysis of dark-pulse capacity to induce a REMS onset

Due to the fact that DPs were automatically delivered, regardless of sleep stage, the probability for a DP to fall within a given state was proportionally related to the time spent in each of the three wake-sleep states from 09:00 to 15:00 $\mathrm{h}$ (i.e. the 6-h period in which DPs were delivered) at the different Tas (Cerri et al., 2005; Franken et al., 1993). At Ta $25^{\circ} \mathrm{C}, 196$ DPs, i.e. the large majority, fell in NREMS, while 57 DPs were delivered during Wake and only 27 during REMS. At Ta $15^{\circ} \mathrm{C}, 133$ DPs took place during NREMS, while 129 fell in Wake and only 18 fell in REMS. In NREMS, DP efficiency (i.e. the number of DPs followed by a REMS onset over the total number of DPs delivered) was significantly higher at $\mathrm{Ta} 25^{\circ} \mathrm{C}(175 / 196$ : 89.3\%) than at Ta $15{ }^{\circ} \mathrm{C}(79 / 133: 59.4 \%, P<0.01)$. On the contrary, DP delivery in Wake was substantially ineffective at both Tas (efficiency: Ta $25^{\circ} \mathrm{C}, 3 / 57: 5.3 \%$; Ta $15^{\circ} \mathrm{C}, 3 / 129$ : $2.3 \%$ ). With respect to the low number of DPs that were delivered during REMS, it was practically impossible to separate the effect of DP from the spontaneous evolution of the REMS episode. For this reason, only the effects of DPs delivered during NREMS will be analysed in this present paper.

The average probability of REMS occurrence (percentage of the number of 4-s epochs in which the animals were in REMS over the total number of 4-s epochs) during the 3-min period which either preceded or followed DP delivery in NREMS, and during the 3-min period in which DP was delivered at either Ta $25^{\circ} \mathrm{C}$ or $\mathrm{Ta} 15^{\circ} \mathrm{C}$, is shown in Fig. 1 . In both conditions, such a probability sharply increased soon after the DP delivery, but it reached higher levels at Ta $25^{\circ}$ than at Ta $15{ }^{\circ} \mathrm{C}$. The median value of the latency for REMS onset after DP delivery was significantly lower at Ta $25^{\circ} \mathrm{C}(44 \mathrm{~s})$ than at Ta $15{ }^{\circ} \mathrm{C}(60 \mathrm{~s}, P<0.01)$. 


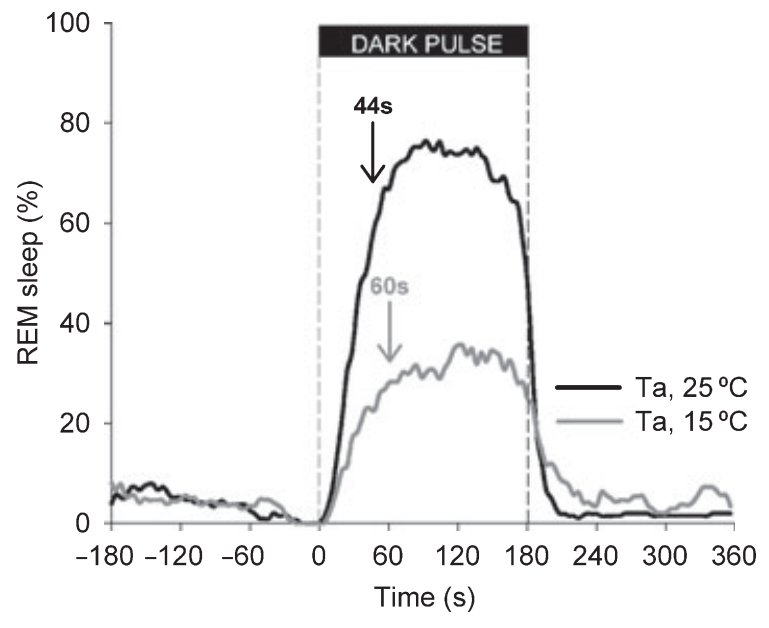

Figure 1. Probability of REM sleep (REMS) occurrence before, during and after dark-pulse (DP) delivery in non-REM sleep (NREMS). The average probability of REMS occurrence (percentage of the number of 4-s epochs in which the animals were in REMS over the total number of 4-s epochs) before, during and after the automatic delivery of a DP (180 s) in NREMS at either Ta $25^{\circ} \mathrm{C}$ (black line) or Ta $15{ }^{\circ} \mathrm{C}$ (grey line) $(n=14$, for each condition) is shown. Time 0 indicates the start of the DP. Data have been calculated with a 4-s resolution. The median latency of the REMS onset is indicated for both conditions.

Time course of EEG and Thy before and during dark-pulse delivery

In Fig. 2, the time course of both the average relative power density in Delta, Sigma and Theta bands of the EEG (mean $\pm \mathrm{SEM}$ ) and the average Thy values $\left({ }^{\circ} \mathrm{C}\right.$, mean \pm SEM) before and during DP delivery (time 0 , start of DP delivery) at either Ta $25^{\circ} \mathrm{C}$ (upper diagram) or Ta $15^{\circ} \mathrm{C}$ (lower diagram) is shown. For each variable, the mean value has been calculated for each animal and data from the different animals have been averaged. Relative power is expressed as the percentage of the average 24-h value under normal laboratory conditions. DPs that were delivered in NREMS were considered for the analysis, independently from their capacity to induce a REMS onset. The selected time window includes the 96-s period that precedes the start of the DP and the 92-s period that follows. Data resolution is $4 \mathrm{~s}$. The bottom part of the graph shows the probability of the animals being in either Wake, NREMS or REMS within each 4-s interval of our analysis. Statistics were performed on $16 \mathrm{~s}$ time windows for the 96-s period that preceded DP delivery.

At low Ta, Delta power and Thy were significantly lower during the 96-s period which preceded the DP with respect to normal laboratory Ta. Soon after the start of the DP, at both Ta $25{ }^{\circ} \mathrm{C}$ and Ta $15{ }^{\circ} \mathrm{C}$, the time course of both Delta, Theta and Sigma power and Thy levels followed the pattern, which characterizes the spontaneous NREMS to REMS transition (Benington et al., 1994; Capitani et al., 2005; Trachsel et al., 1988). At time 0, Delta power starts decreasing, Theta and Sigma power start increasing, and Thy is maintained at stable levels for a few seconds before rising.
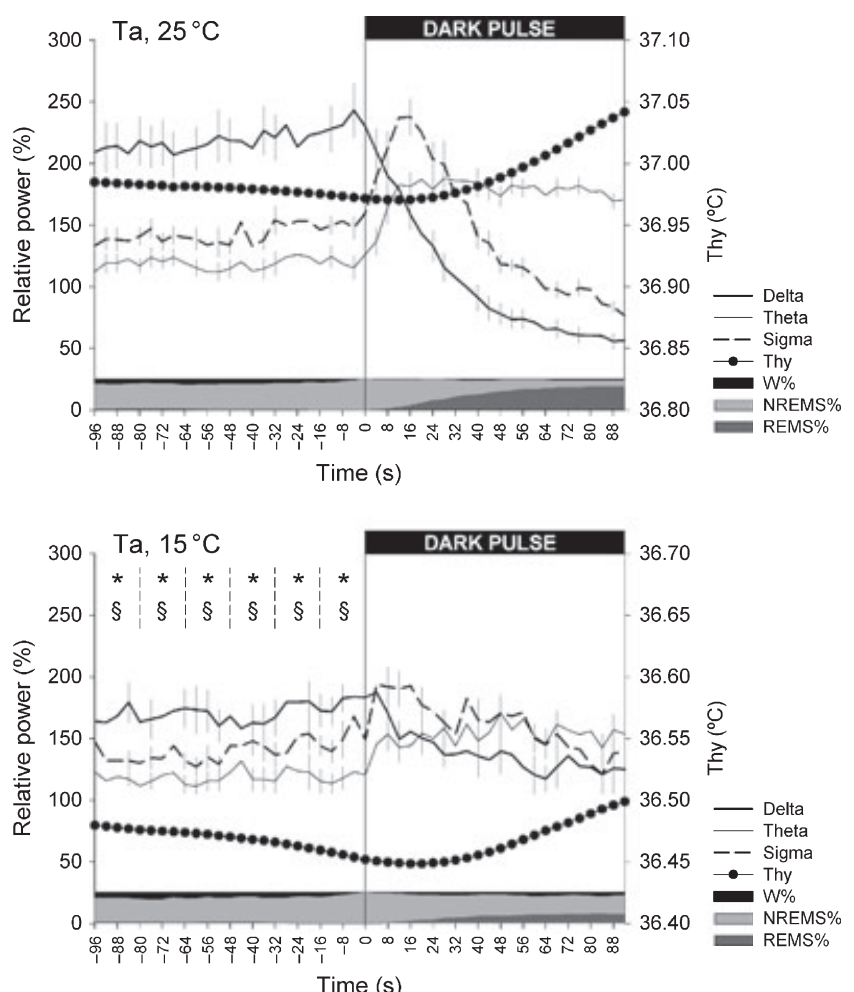

Figure 2. Time course of EEG power and hypothalamic temperature (Thy) before and during dark-pulse (DP) delivery in non-REM sleep (NREMS). The time course (4-s epochs, $n=14$ ) of the average power density (left axis) in the Delta $(0.75-4.0 \mathrm{~Hz}$; thick), Theta (5.5-9.0 Hz; thin) and Sigma $(11.0-16.0 \mathrm{~Hz}$; dashed) bands of the EEG and average Thy $\left({ }^{\circ} \mathrm{C}\right.$, right axis; filled circles) during the $96-\mathrm{s}$ period that precedes and the 92-s period that follows the automatic delivery of a dark pulse (DP, 0 s) in NREMS at either Ta $25^{\circ} \mathrm{C}$ (upper diagram) or $\mathrm{Ta} 15^{\circ} \mathrm{C}$ (lower diagram) is shown. Average power density is expressed as the percentage of the average 24-h levels $( \pm$ SEM) for each EEG frequency band. For a better visual quality of the diagram, SEM is not shown for Thy. Statistical significant differences between the two ambient conditions in the 96-s period that precedes DP delivery are shown (16-s sequences; $P<0.05$; *, Delta power; $\S$, Thy). The bar over the x-axis, whose total height represents $100 \%$, shows the average percentage of probability for the animal to be in Wake (W, black), non-REM sleep (NREMS, light grey), or REM sleep (REMS, dark grey) during each 4-s epoch.

The time course of the former variables has been further analysed in order to compare, at either Ta $25^{\circ} \mathrm{C}$ (Fig. 3) or Ta $15^{\circ} \mathrm{C}$ (Fig. 4), DPs which led to a REMS onset (effective DP, right) with those that did not (ineffective DP, left). Only 12 animals at Ta $25^{\circ} \mathrm{C}$ and 13 animals at Ta $15^{\circ} \mathrm{C}$ were used for analysis, due to the lack of ineffective DPs in two animals at Ta $25^{\circ} \mathrm{C}$ and in one animal at Ta $15{ }^{\circ} \mathrm{C}$.

At Ta $25^{\circ} \mathrm{C}$, a different time course for EEG powers and Thy was observed between effective and ineffective DPs during both the 96-s period preceding the DP and the 92-s period during DP delivery. In the 96-s period that preceded the ineffective DPs, the power density in the different EEG band (Delta, Theta and Sigma) was significantly depressed in the 

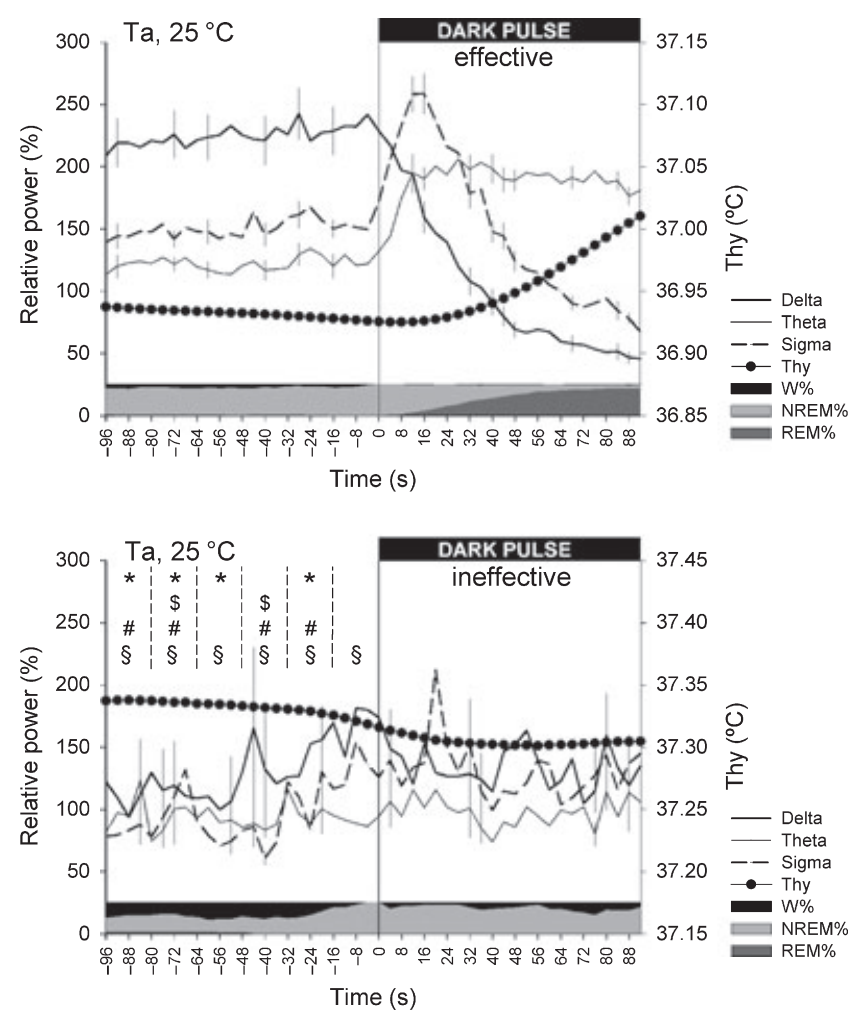

Figure 3. Time course of EEG power and hypothalamic temperature (Thy) at Ta $25^{\circ} \mathrm{C}$, before and during dark-pulse (DP) delivery in nonREM sleep (NREMS). The time course (4-s epochs, $n=12$ ) of the average power density (left axis) in the Delta $(0.75-4.0 \mathrm{~Hz}$; thick), Theta $(5.5-9.0 \mathrm{~Hz}$; thin) and Sigma $(11.0-16.0 \mathrm{~Hz}$; dashed) bands of the EEG and average Thy $\left({ }^{\circ} \mathrm{C}\right.$, right axis; filled circles) during the $96-\mathrm{s}$ period that precedes and the 92 -s period that follows the automatic delivery of a DP $(0 \mathrm{~s})$ in NREMS at Ta $25^{\circ} \mathrm{C}$, for DPs that led to a REM sleep (REMS) onset (effective DPs: upper diagram) or were ineffective (lower diagram) is shown. Average power density is expressed as the percentage of the average 24-h levels ( \pm SEM) for each EEG frequency band. For a better visual quality of the diagram, SEM is not shown for Thy. Statistical significant differences between the two conditions in the 96-s period that precedes DP delivery are shown (16-s sequences; $P<0.05$; *Delta power; $\$$, Theta power; , Sigma power; $\S$, Thy). The bar over the $\mathrm{x}$-axis, whose total height represents $100 \%$, shows the average percentage of probability for the animal to be in Wake (W, black), NREMS (light grey), or REMS (dark grey) during each 4-s epoch.

majority of the 16-s intervals, while Thy levels were significantly higher in ineffective DPs than in effective DPs. Moreover, Wake appeared to be a more probable state in the 96-s period that preceded ineffective DPs. In the 92-s period which followed the start of the ineffective DP, no detectable changes were present in the time course of the power densities for the different EEG bands, while Thy did not show any increasing trend. At $\mathrm{Ta} 15^{\circ} \mathrm{C}$, in the 96 -s period which preceded DP delivery only Sigma power appeared to be significantly lower for ineffective DPs with respect to effective DPs, although statistically significance was reached only in two 16-s time windows. Also, a decreasing trend in Thy was observed. In the 92-s period which followed the effective DPs,
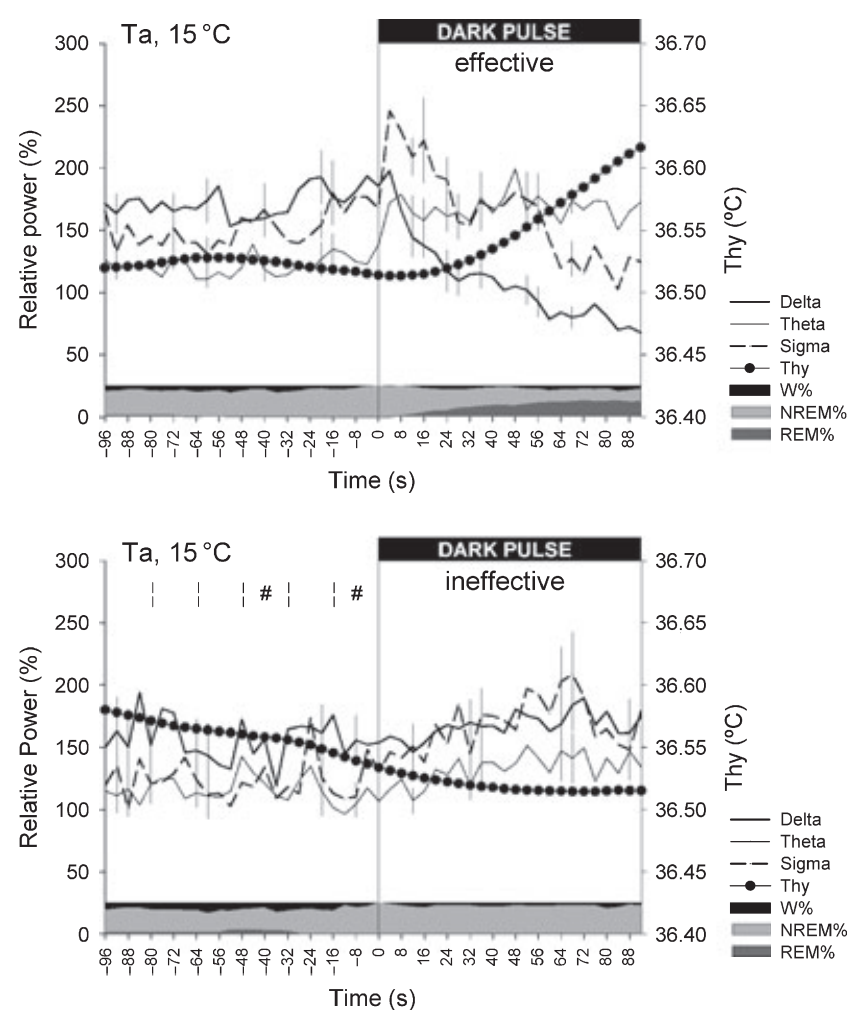

Figure 4. Time course of EEG power and hypothalamic temperature (Thy) at $\mathrm{Ta} 15^{\circ} \mathrm{C}$, before and during dark-pulse (DP) delivery in non-REM sleep (NREMS). The time course (4 s epochs, $n=13$ ) of the average power density (left axis) in the Delta $(0.75-4.0 \mathrm{~Hz}$; thick), Theta $(5.5-9.0 \mathrm{~Hz}$; thin) and Sigma $(11.0-16.0 \mathrm{~Hz}$; dashed) bands of the EEG and average Thy $\left({ }^{\circ} \mathrm{C}\right.$, right axis; filled circles) during the 96-s period that precedes and the 92-s period that follows the automatic delivery of a DP $(0 \mathrm{~s})$ in NREMS at Ta $15^{\circ} \mathrm{C}$, for DPs that led to a REM sleep (REMS) onset (effective DPs: upper diagram) or were ineffective (lower diagram) is shown. Average power density is expressed as the percentage of the average 24-h levels $( \pm$ SEM) for each EEG frequency band. For a better visual quality of the diagram, SEM is not shown for Thy. Statistical significant differences between the two conditions in the 96-s period that precedes DP delivery are shown (16-s sequences; $P<0.05$; \#, Sigma power The bar over the $\mathrm{x}$-axis, whose total height represents $100 \%$, shows the average percentage of probability for the animal to be in Wake (W, black), NREMS (light grey), or REMS (dark grey) during each 4-s epoch.

the dynamics of both EEG powers and Thy resembled those observed for effective DPs at Ta $25^{\circ} \mathrm{C}$, while ineffective DPs were characterized by an increasing trend in Theta and Sigma power and by a constant fall in Thy.

\section{Effects on wake-sleep stages}

The tonic effects of different treatments on the amount of Wake, NREMS and REMS (min, mean \pm SEM) are shown in Fig. 5. Data are shown for both the first 6-h period (09:0015:00 $\mathrm{h}$ ) and the subsequent 18-h period (15:00-09:00 $\mathrm{h}$ ) of the day.

The amount of Wake was largely affected by the exposure to low Ta and to a lesser extent by DP delivery. This amount 

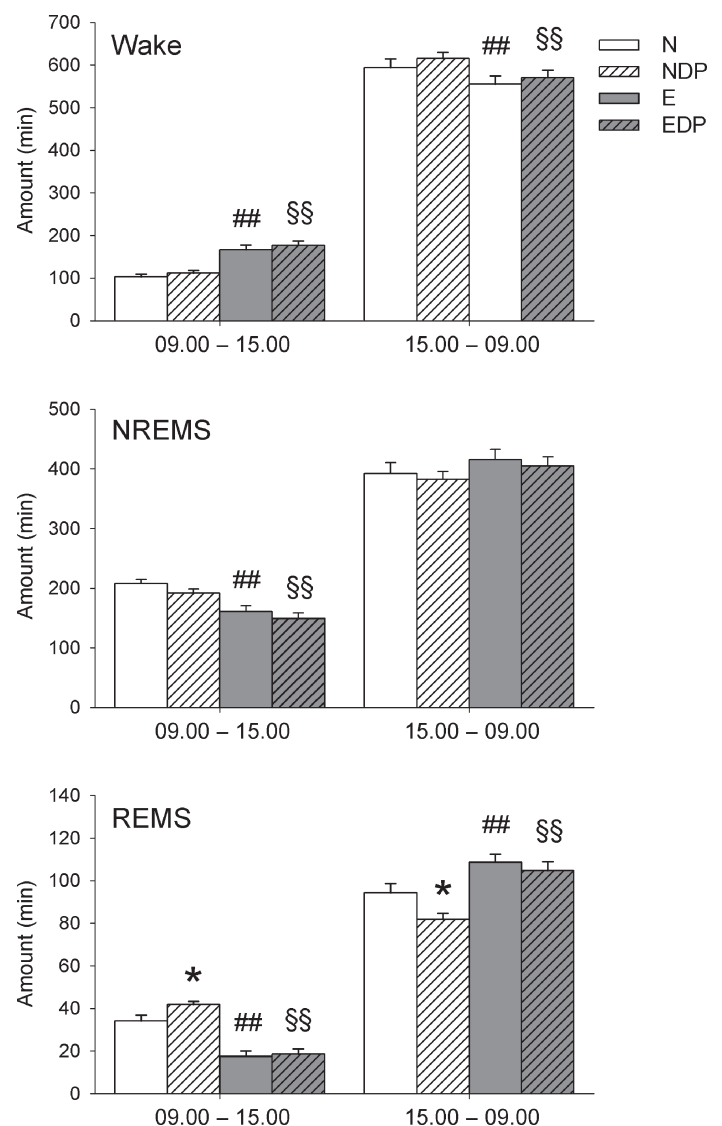

Figure 5. Time spent in different sleep stages under different environmental influences. The total amount (min, mean \pm SEM, $n=14$ ) of Wake, non-REM sleep (NREMS) and REM sleep (REMS), during the first 6-h period (09:00-15:00 h) and the subsequent 18-h period (15:0009:00 h) of the day, under the following experimental conditions is shown: normal laboratory $\mathrm{Ta}\left(\mathrm{Ta} 25^{\circ} \mathrm{C}, \mathrm{N}\right.$, empty); dark-pulse delivery at normal laboratory Ta (Ta $25^{\circ} \mathrm{C}$, NDP, empty dashed); exposure to low Ta (Ta $15^{\circ} \mathrm{C}, \mathrm{E}$, grey); dark-pulse delivery at low Ta (Ta $15^{\circ} \mathrm{C}$, EDP, grey dashed). Dark-pulse delivery and exposure to low Ta occurred only during the first 6-h period. Statistical significance of post hoc individual comparisons is shown (*, NDP versus N; , E versus N; $\S$, EDP versus NDP; $P<0.05$, one symbol; $P<0.01$, two symbols).

increased in the first 6-h period of exposure and decreased in the following 18-h period in both experimental conditions in which animals were exposed to low Ta (E, EDP) with respect to those in which they were kept at normal laboratory temperature (N, NDP), while a slight increase was observed in the whole 24-h period (min, mean \pm SEM: $\mathrm{N}$, $696.9 \pm 25.8 ; \mathrm{NDP}, 727.2 \pm 11.2 ; \mathrm{E}, 722.2 \pm 24.8$; EDP, $747.3 \pm 20.8$ ). A small increase in the amount of Wake in each hourly block was also observed in conditions in which DPs were delivered, at both Ta levels. A two-way Anova shows that the effects of Ta lowering are statistically significant both in the 6-h exposure period, in the following 18-h period at normal laboratory Ta, and in the 24-h period $(6 \mathrm{~h}: P<0.01 ; 18 \mathrm{~h}$ : $P<0.01 ; 24$ h: $P<0.05)$. The wake-enhancing effect of DP delivery reaches statistical significance only when the whole 24h period is considered $(P<0.05)$. Post hoc individual com- parison within homologous DP delivery conditions shows the presence of statistically significant differences either in the 6-h period (N versus E: $P<0.01$; NDP versus EDP: $P<0.01$ ) or in the following 18 -h period (N versus E: $P<0.01$; NDP versus EDP: $P<0.01$ ).

The exposure to low Ta, not DP delivery, affected the amount of NREMS. The amount of NREMS decreased in the first 6-h period of exposure and increased in the following 18-h period in both experimental conditions in which animals were exposed to low Ta (E, EDP) with respect to those in which they were kept at normal laboratory temperature (N, NDP), while a small decrease was observed in the whole 24-h period (min, mean \pm SEM: N, $599.6 \pm 23.4$; NDP, $574.1 \pm 10.5$; E, $576.6 \pm 22.0$; EDP, $554.2 \pm 18.4$ ). A two-way ANova shows that the effects of Ta lowering are statistically significant in the 6-h exposure period, in the following 18-h period at normal laboratory Ta, and in the whole 24 -h period $(6 \mathrm{~h}: P<0.01$; 18 h: $P<0.01 ; 24$ h: $P<0.05)$. Post hoc individual comparison within homologous DP delivery conditions shows the presence of statistically significant differences only in the 6-h period (N versus E: $P<0.01$; NDP versus EDP: $P<0.01$ ).

The amount of REMS was influenced by both the exposure to low Ta and DP delivery. Such an amount was depressed during exposure to low Ta (E) with respect to $\mathrm{N}$, while a REMS rebound was observed during the following recovery period at normal laboratory Ta. The influence of DP delivery on the amount of REMS was strongly dependent on Ta levels: at Ta $25^{\circ} \mathrm{C}$ (NDP), an increase during the first 6-h period and a decrease during the following 18 -h period was observed with respect to $\mathrm{N}$ levels, while at $\mathrm{Ta} 15^{\circ} \mathrm{C}$ (EDP) REMS amount was scarcely affected. No relevant differences in the amount of REMS were present among the four experimental conditions when the whole 24-h amount was taken into account (min, mean \pm SEM: $\mathrm{N}, \quad 128.6 \pm 5.7 ; \quad \mathrm{NDP}, 123.8 \pm 3.5 ; \quad \mathrm{E}$, $126.2 \pm 4.0$; EDP, $123.6 \pm 3.7$ ). A two-way ANOva shows that both the effects of Ta lowering and those of DP delivery are statistically significant in both the first 6-h period (Ta, $P<0.01$; DP delivery, $P<0.05)$ and in the following 18 -h period (Ta, $P<0.01$; DP delivery, $P<0.05$ ). However, in the first 6 -h period a statistically significant interaction is present between the two factors $(P<0.05)$. The effects due to Ta lowering are statistically significant even when post hoc individual comparisons are made within homologous DP delivery conditions (N versus E: $6 \mathrm{~h}, P<0.01 ; 18 \mathrm{~h}$ $P<0.01 ; \quad$ NDP versus EDP: 6 -h, $P<0.01 ; 18$-h, $P<0.01$ ), while the effects of DP delivery reach statistical significance only at normal laboratory temperature conditions (N versus NDP: $6 \mathrm{~h}, P<0.05 ; 18 \mathrm{~h}, P<0.05$ ).

In Fig. 6, the effect of the different treatments on the relative delta power density in NREMS (mean \pm SEM, $100 \%=$ average 24 -h power at normal laboratory conditions) during the whole 24-h period is shown. Data are shown with a 2-h resolution. Delta power appears to be affected in a complex way by either the exposure to low Ta or DP delivery. This complexity was emphasized by the statistical analysis of the results. In particular, as a first step, a three-way repeated 

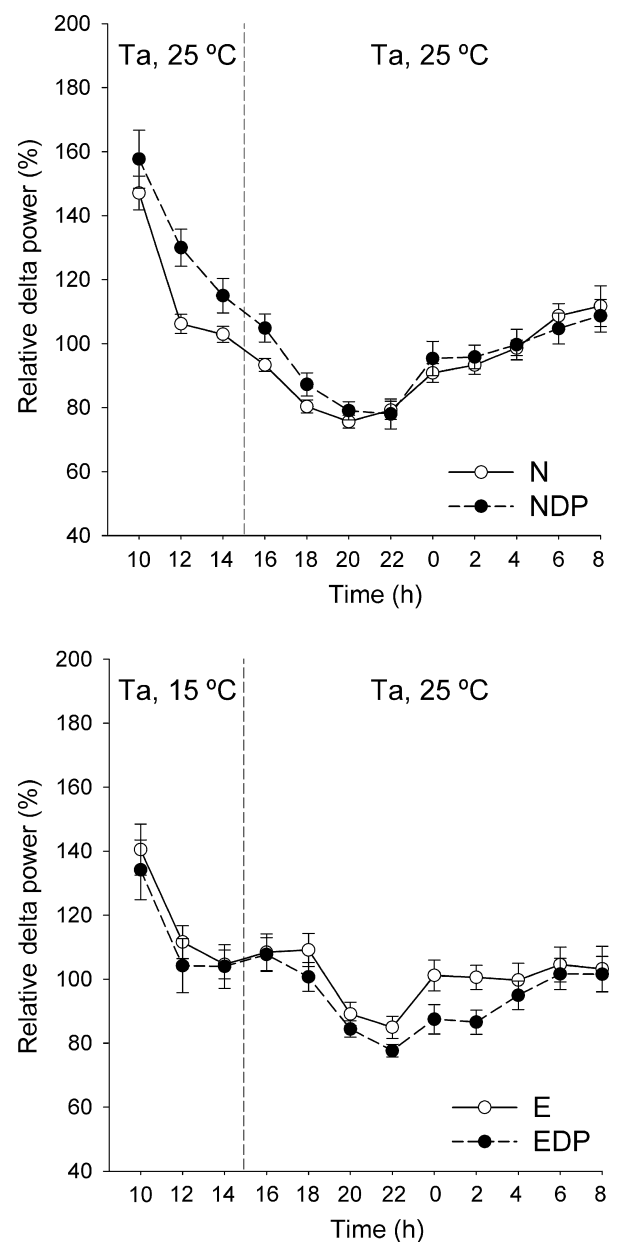

Figure 6. Time course of Delta power under different environmental influences. The time course (2-h interval, $n=14$, the midpoint of each 2-h interval is shown) of the average power density in the Delta band $(0.75-4.0 \mathrm{~Hz})$ of the EEG during non-REM sleep (NREMS) under the following experimental conditions: Normal laboratory Ta $\left(\right.$ Ta $25^{\circ} \mathrm{C}$, $\mathrm{N}$; empty) and dark-pulse delivery at normal laboratory Ta $\left(\right.$ Ta $25^{\circ} \mathrm{C}$, NDP; filled), upper diagram; exposure to low Ta (Ta $15{ }^{\circ} \mathrm{C}$, E; empty) and dark-pulse delivery at low Ta (Ta $15{ }^{\circ} \mathrm{C}$, EDP; filled), lower diagram. Average power density is expressed as the percentage of the average 24 -h levels $( \pm$ SEM). The dashed vertical line indicates the limit of the first 6-h period (09:00-15:00 h) during which dark-pulse delivery and exposure to low Ta occurred.

measure ANOVA was carried out, in which not only factors 'Ta' and 'DP', but also factor 'time' (12 levels, corresponding to each of the different 2-h blocks), were taken into account. The results of the analysis showed that all interactions were statistically significant $(\mathrm{Ta} \times \mathrm{DP}, \quad P<0.05 ; \quad \mathrm{DP} \times$ time, $P<0.05$; Ta $\times$ time, $P<0.01$; Ta $\times \mathrm{DP} \times$ time, $P<0.05$ ). On this basis, for a better statistical assessment of both the main effects and Ta $\times$ DP interaction at each level of factor 'time', a separate two-way ANOva was carried out for each 2-h block. Three main effects are visible: (i) during the first 6-h period, an increase in Delta power was observed when animals kept at Ta $25{ }^{\circ} \mathrm{C}$ were stimulated with DPs. Such a DP enhancing effect was not present at Ta $15{ }^{\circ} \mathrm{C}$. A two-way Anova shows that the effect related to factor $\mathrm{Ta}$ is significant at either
10:00 h $(P<0.05)$ or $12: 00 \mathrm{~h}(P<0.01)$, but a significant interaction between the two treatments is observed at 10:00 h $(P<0.05), 12: 00 \mathrm{~h}(P<0.05)$, or $14: 00 \mathrm{~h}(P<0.05)$. Post hoc individual comparisons show that Delta power during the NDP condition is significantly higher with respect to either N $(12: 00 \mathrm{~h}, P<0.05)$ or EDP $(10: 00 \mathrm{~h}, P<0.05 ; 12: 00 \mathrm{~h}$, $P<0.05)$; (ii) in the first 6-h period $(15: 00-21: 00 \mathrm{~h})$, which followed the exposure to low Ta, an increase in Delta power was observed with respect to the normal laboratory temperature conditions independently of DP delivery. A two-way ANOva shows that the effect related to factor Ta reaches statistical significance at either 18:00 h $(P<0.01)$ or $20: 00 \mathrm{~h}$ $(P<0.01)$, while a significant interaction is present at either 16:00 h $(P<0.05)$ or 18:00 h $(P<0.05)$. Post hoc individual comparisons show that Delta power is significantly higher in condition E than in condition $\mathrm{N}(18: 00 \mathrm{~h}, P<0.01 ; 20: 00 \mathrm{~h}$, $P<0.01$ ); (iii) during the central part of the recovery period (i.e. the first $6 \mathrm{~h}$ of darkness, 21:00-03:00 h) a decrease in Delta power was observed when animals were stimulated with DPs while they were kept at $\mathrm{Ta} 15^{\circ} \mathrm{C}$. The effect was absent when the rat was kept at Ta $25^{\circ} \mathrm{C}$. A two-way Anova shows that the effect of the factor DP reaches statistical significance at either 22:00 h $(P<0.05)$ or $02: 00 \mathrm{~h}(P<0.05)$, while a significant interaction is present at either $00: 00 \mathrm{~h}(P<0.05)$ or $02: 00 \mathrm{~h}(P<0.01)$. Post hoc individual comparisons show that Delta power in EDP condition is significantly lower than in either $\mathrm{E}(00: 00 \mathrm{~h}, P<0.05 ; 02: 00 \mathrm{~h}, P<0.01)$ or NDP condition $(02: 00$ h $P<0.05)$.

\section{Experiment II}

Analysis of dark-pulse capacity to induce a REMS onset

The manual delivery of DPs during selected NREMS episodes did not substantially change DP efficiency and the latency of REMS onset with respect to what was observed in experiment I at either Ta $25^{\circ} \mathrm{C}$ or Ta $15{ }^{\circ} \mathrm{C}$. DP efficiency in inducing a REMS onset at $\mathrm{Ta} 25^{\circ} \mathrm{C}(72 / 79$ : $91.1 \%)$ was significantly higher than that observed at Ta $15{ }^{\circ} \mathrm{C}(36 / 73$ : $49.3 \%$; $P<0.01$ ). The median value of the latency for REMS onset after the start of the DP was significantly shorter at Ta $25^{\circ} \mathrm{C}$ (33 s) than at $\mathrm{Ta} 15^{\circ} \mathrm{C}(76 \mathrm{~s}, P<0.01)$.

Time course of EEG, Thy and EKG before and during dark-pulse delivery

The time course of the average power density in Delta, Sigma and Theta bands of the EEG and average Thy values before and during DP delivery at either Ta $25^{\circ} \mathrm{C}$ or Ta $15^{\circ} \mathrm{C}$ is not shown, due to the absence of qualitative difference from what was observed in experiment I, under both ambient conditions. As in experiment $\mathrm{I}$, at $\mathrm{Ta} 25^{\circ} \mathrm{C}$ both a decrease in Delta power and a rise in Thy were observed in the 96-s period that preceded the delivery of ineffective DPs with respect to effective ones. However, these differences did not reach statistical significance. 

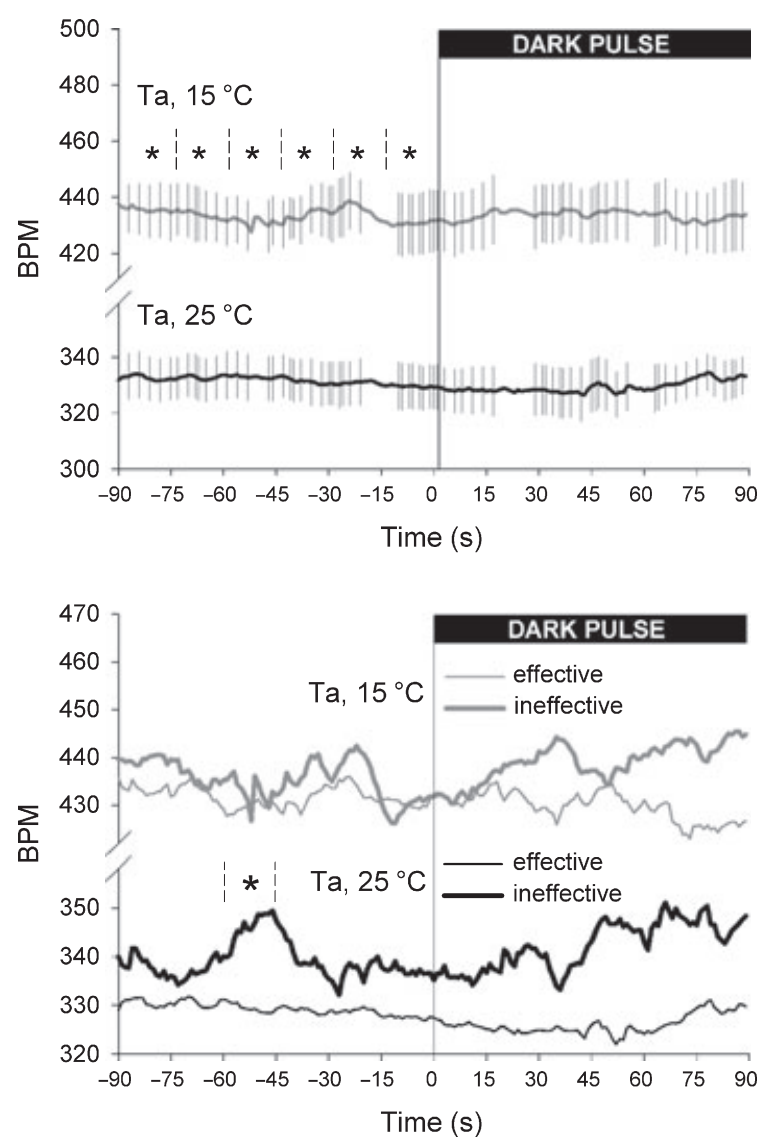

Figure 7. Time course of heart rate before and during dark-pulse (DP) delivery in non-REM sleep (NREMS). The time course (1-s resolution, $n=6$ ) of the average heart rate (BPM \pm SEM) during the 90 -s period that precedes the manual delivery of a DP $(0 \mathrm{~s})$ and the 90 -s period that follows during NREMS is shown. In the upper diagram, the time course related to DPs delivery at either Ta $15^{\circ} \mathrm{C}$ (grey line) or Ta $25^{\circ} \mathrm{C}$ (black line) is shown. Statistical significant differences between the two ambient conditions in the 90 -s period that precedes DP delivery are shown $(15$-s sequences; $*, P<0.05)$. In the lower diagram, the time course related to effective DPs is shown separately from that of ineffective DPs at either Ta $15^{\circ} \mathrm{C}$ (grey: effective, thin; ineffective, thick) or Ta $25^{\circ} \mathrm{C}$ (black: effective, thin; ineffective, thick). For a better visual quality of the lower diagram, SEM is not shown. Statistical significant differences between effective and ineffective DPs in the 90 -s period that precedes DP delivery are shown (15-s sequences; $*, P<0.05)$.

In Fig. 7, the average time course of heart rate (bpm, mean \pm SEM) before and during DP delivery (time 0 , start of DP delivery) at either Ta $25^{\circ} \mathrm{C}$ (upper diagram) or Ta $15^{\circ} \mathrm{C}$ (lower diagram) is shown. In the upper diagram, all DPs that were delivered in NREMS have been considered for the analysis, independently of their efficacy in inducing REMS. In the lower diagram, the DPs which led to a REMS onset (effective DPs) have been compared with those that did not (ineffective DP). For each variable, the mean value has been calculated for each animal and data from the different animals have been averaged. The selected time window includes the 90s period that precedes the start of the dark pulse and the 90-s period that follows it. Data resolution is $1 \mathrm{~s}$, and heart rate was calculated as the ratio between 60 and the average $\mathrm{R}-\mathrm{R}$ interval duration (s) within each second. Statistics have been performed on six 15-s time windows for the 90 -s period that preceded the DP delivery. Heart rate was tonically higher at Ta $15^{\circ} \mathrm{C}$ than at Ta $25^{\circ} \mathrm{C}$. At Ta $15{ }^{\circ} \mathrm{C}$, in the 90 -s period which preceded DP delivery, heart rate appeared to be higher and much less stable for ineffective DPs than for effective ones and statistical significance is reached in the $-60 \mathrm{~s}$ to $-45 \mathrm{~s}$ time window. At Ta $15^{\circ} \mathrm{C}$, no clear differences in the time course were present between effective and ineffective DPs.

\section{DISCUSSION}

The main findings of the present study can be summarized as follows: (i) the well-known capacity of a brief DP delivery to induce a REMS onset in the albino rat with a high probability and a short latency (Lisk and Sawyer, 1966; Rechtschaffen et al., 1969) was impaired when the animal was kept al low Ta to a degree that is comparable to that of the spontaneous depression in REMS occurrence; (ii) the DP delivery protocol induced tonic changes in REMS occurrence during both the 6$\mathrm{h}$ period of delivery and the following $18 \mathrm{-h}$ period at Ta $25^{\circ}$, but not at Ta $15^{\circ} \mathrm{C}$. Both aspects suggest that the efficacy of DPs at low Ta was dampened by the overall neurophysiologic and autonomic state of the animal resulting from central thermoregulatory activation. The incapacity of DP to overcome this hindrance underlines the crucial physiological meaning of the mutual exclusion of thermoregulatory activation and REMS occurrence. On this basis, DP capacity to induce REMS will be discussed within the frame of the challenging interaction between the regulations of body temperature and REMS.

\section{Dark-pulse capacity to induce a REMS onset at normal laboratory Ta}

The analysis of the time course of power densities in the three different bands of the EEG and Thy levels immediately before and during DP delivery, showed that, at normal laboratory Ta, DP delivery during NREMS is able to induce with a very short latency the EEG and autonomic changes which characterize the NREMS to REMS transition in the rat. NREMS to REMS transition has been deeply analysed in different species with respect to either the pattern of EEG (Benington et al., 1994; Capitani et al., 2005; Gottesmann, 1996; McCarley and Hobson, 1970; Trachsel et al., 1988) or that of autonomic activity (Capitani et al., 2005; Parmeggiani et al., 1975). In particular, in the rat the time course of the EEG power appears to be stereotyped (Benington et al., 1994; Capitani et al., 2005; Trachsel et al., 1988) and it is characterized by a decrease in Delta power and a parallel increase in Sigma and Theta power which occur within a time interval of 30-60 s before the beginning of the REMS episode.

The present data show that the changes in the EEG power in the NREMS to REMS transitions that were triggered by a DP were not qualitatively different from those observed in 
spontaneous transitions. Moreover, the average duration (44 s) of the triggered NREMS to REMS transition that led to REMS onset was within the range of the spontaneous transition (30-60 s) (Benington et al., 1994; Capitani et al., 2005; Trachsel et al., 1988). Furthermore, the time course of an index of autonomic activity, such as the hypothalamic temperature (Parmeggiani, 2007), did not seem to show any relevant difference from the time course which precedes the occurrence of a spontaneous REMS episode (Capitani et al., 2005). In fact, Thy appears to reverse its decreasing trend soon after the DP delivery, starting to ascend after the first $12 \mathrm{~s}$ from the start of the DP (see Fig. 3, upper diagram). In a previous experiment from our laboratory (Capitani et al., 2005), a small increase in Thy during the spontaneous transition was shown to be associated with a high probability for the transition to be followed by a consolidated REMS episode, while a small decrease in Thy usually characterizes an unsuccessful transition, leading to an awakening.

The DP triggers a transition to REMS that does not apparently differ from a spontaneous one from either an EEG or an autonomic point of view. This would suggest a direct facilitating action via a retinal efferent pathway on the neural network physiologically involved in REMS onset. Pioneering experiments attempted to define the neuroanatomical pathways by which the sudden light-off exerts its effects. These experiments showed that a lesion of the primary optic tract, but not that of either the accessory optic system or the retinohypothalamic tract at the levels of the suprachiasmatic nucleus (SCN) (Sisk and Stephan, 1982), was able to attenuate the capacity of short LD cycles to concentrate REMS during lightoff periods (Borbély, 1978; Johnson et al., 1970). Further lesion studies targeting the primary optic system have shown that DP efficacy was reduced following wide lesions of PT, but not of superior colliculus (SC) or visual cortex (Miller et al., 1998, 1999). Since direct projections from PT to either laterodorsal tegmental nucleus (LDT) or peduncolopontine nucleus (PPT), two of the key areas in REMS regulation (Steriade and Mc Carley, 1990) have been shown (Cornwall et al., 1990; Terenzi et al., 1995), it has been proposed that the DP effect is mediated by a retinal efferent pathway through PT, directly targeting the REMS regulating network within the brain stem. Interestingly, the response to DP delivery has been shown to be strengthened by dark-rearing (Prichard et al., 2004), a condition that apparently reduces the distribution of retinal fibres to $\mathrm{SC}$ but not to PT or the hypothalamus (Prichard et al., 2007).

However, the involvement of different sub-cortical structures should be taken into account. With respect to the 'circadian visual system', enhanced retinal projections to the controlateral intergeniculate leaflet (IGL) have been recently shown in albino rats by comparison with pigmented animals (Fleming et al., 2006). IGL is reciprocally connected with SCN and directly projects to both LDT/PPT and to portions of the PO-AH (Morin and Allen, 2006) that have been shown to be involved in REMS regulation (Lu et al., 2000, 2002). Also, direct projection from the retina to the dorsal raphe nucleus
(DRN), an area that is known to be involved in REMS regulation (Brown and Mc Carley, 2005), have been described (Fite et al., 1999). DRN serotonergic neurons have been suggested to synapse directly with cholinergic neurons within LDT (Wang et al., 2000).

In very few cases, DP delivery in NREMS was unable to induce a REMS onset at Ta $25{ }^{\circ} \mathrm{C}$. However, due to the small number of ineffective DPs, it is difficult to get robust data on which a comparison with successful transitions to REMS can be carried out. In experiment I (automatic delivery), the DP appeared to be unable to induce a REMS onset when it was delivered during apparently not fully consolidated NREMS episodes, which were characterized by a low Delta power density and by a relatively higher Thy level with respect to episodes in which DPs were effective. Also in experiment II (manual delivery, data not shown), Delta power and Thy appeared to be lower and higher, respectively, in ineffective DPs with respect to effective DPs, but in both cases the difference was not statistically significant, possibly due to either the lower number of animals or the lower number of DPs delivered to each animal in experiment II with respect to experiment I. However, in experiment II a difference in the time course of heart rate was observed between effective and ineffective DPs, since the heart rate in the time period preceding the ineffective DP delivery was higher and less stable than when DPs were effective. Despite such an increase being statistically significant only during a 15 -s time window which preceded DP delivery, higher levels in both Thy and heart rate would suggest that ineffective DPs were delivered in a moment of rather high sympathetic activity, i.e. a condition not favourable for REMS occurrence (Parmeggiani, 2005, 2007).

\section{Dark-pulse capacity to induce a REMS onset at low Ta}

DP efficiency in inducing an REMS onset largely decreased during exposure to low ambient temperature. Such an impairment was accompanied by a decrease in the total amount of REMS to about $50 \%$ of that observed at normal laboratory conditions. An even sharper decrease occurred when the experimental sessions in which DP delivery was carried out at different Ta were compared. DP efficiency was depressed to an extent that was comparable with that observed in spontaneous REMS occurrence. It is worth noting that DPs were not delivered during the first hour of exposure, when the effect on spontaneous REMS occurrence was the largest.

Thermoregulation and REMS have been shown to interact in different species and, in particular, cold exposure is known to depress spontaneous REMS occurrence (Heller, 2005; Parmeggiani, 2003). This may be a consequence of the thermoregulatory impairment that has been shown to occur during REMS in different species and that is thought to be due to a change in the activity of the central thermostat at the preoptic-hypothalamic level (Parmeggiani, 2003, 2005). On this basis, it has been hypothesized that the continuous 
thermoregulatory drive induced by the exposure to low Ta opposes the drive leading to REMS occurrence (Parmeggiani, 2003). Consequently, the activity of the thermoregulatory neuronal network would be able to interfere with the activity of the network that leads to REMS onset.

These two networks would apparently overlap at $\mathrm{PO}-\mathrm{AH}$ level. Single-unit recording experiments have shown that POAH neurons, and in particular cold-sensitive neurons, strongly reduce their thermal sensitivity during REMS (Alam et al., 1995; Parmeggiani, 1987). Moreover, PO-AH cellular activity has been shown to be related to REMS occurrence in rats kept under different environmental conditions (Amici et al., 2005; Perez et al., 1991; Zamboni et al., 2004). Finally, a group of cells whose activity appears to be critically related with REMS occurrence has been found in both the ventral lateral preoptic area (Lu et al., 2000, 2002) and in the median preoptic nucleus (Gvilia et al., 2006). Since PO-AH has been shown to be in direct connection with the neuronal network which leads to REMS onset within the brainstem (Simerly and Swanson, 1988), it may be suggested that the hypothalamic thermoregulatory centres act as a higher hierarchical level controller over the brainstem network through a direct descending pathway, and that cold-induced changes in the activity of $\mathrm{PO}-\mathrm{AH}$ neurons depress the facilitating effect on this network induced by the retinal efference. The results of the present study therefore support previous data suggesting that $\mathrm{PO}-\mathrm{AH}$ is a crucial component of the neural network involved in the regulation of REMS occurrence (Szymusiak et al., 2007; Zamboni et al., 2004).

Also, there is evidence of projections from the $\mathrm{PO}-\mathrm{AH}$ to the orexin neuronal field in the lateral hypothalamus (LH) in the rat (Yoshida et al., 2006). Orexin mRNA has been shown to increase in LH following acute cold exposure in this species (Ida et al., 2000). Moreover, in the anesthetized rat the capacity to induce a REMS-like episode by microinjecting carbachol, a cholinergic agonist, into the pontine tegmentum is suppressed by the activation of the orexinergic system in LH (Lu et al., 2007). Finally, it cannot be overlooked that a dense retinal afference to the PO-AH either directly (Levine et al., 1991) or through the IGL and SC (Morin and Allen, 2006) has been shown. This indicates not only the possibility of a direct involvement of PO-AH in the control of REMS expression under the influence of either rhythmical (DP, short LD cycles) (Borbély, 1978; Johnson et al., 1970) or tonic (continuous darkness) (Borbély, 1978) darkness, but also that DP signaling and thermoregulation might directly interfere at PO-AH levels.

The analysis of the time course of the power in the different EEG bands and of Thy levels in the time period which preceded DP delivery, shows that when DPs were automatically delivered at $\mathrm{Ta} 15^{\circ} \mathrm{C}$ they fell during a NREMS episode that was less consolidated than at $\mathrm{Ta} 25^{\circ} \mathrm{C}$ and in which Delta power was lower than at Ta $25^{\circ} \mathrm{C}$. However, the importance of NREMS consolidation during DP delivery in permitting REMS induction is challenged by the fact that the efficacy of DP was not increased at Ta $15^{\circ} \mathrm{C}$ under manual
DP delivery, when NREMS consolidation was the main criteria when switching off the light. Similarly, the importance of Delta power levels in permitting REMS induction is challenged by the fact that no significant differences in Delta power levels were observed between effective and ineffective DPs at Ta $15{ }^{\circ} \mathrm{C}$. Thus, it appears to be more relevant that the exposure to low ambient temperature induced a tonic increase in sympathetic activity, as evidenced by the tonic increase in heart rate, which may interfere with the occurrence of REMS as previously discussed (Parmeggiani, 2005, 2007).

\section{Tonic effects of dark-pulse delivery on REMS and NREMS}

The DP delivery protocol used in experiment I also led to tonic changes in the wake-sleep states during either the 6-h period in which the DPs were delivered or the following 18-h period. Data from experiment I show that, at normal laboratory temperature, automatic and random DP delivery induced an increase in the amount of REMS within the 6-h period in which the DPs were delivered, followed by an apparently compensatory negative rebound in the following 18 -h period. In previous experiments in other laboratories using light-off delivery protocols (Benca et al., 1998; Rechtschaffen et al., 1969), the daily duration of darkness was maintained constant so as not to modify the $12 \mathrm{~h}: 12 \mathrm{~h}$ LD balance in the $24-h$ period. In these experiments, an increase in the REMS amount observed when the light was off was apparently compensated by a reduction in the amount of REMS in the time periods between subsequent DPs, when the light was on. This was not the case in the present experiment, where the amount of REMS was increased during the 6-h DP delivery period at Ta $25^{\circ} \mathrm{C}$. Since the DP delivery protocol consisted of 20 pulses of 3 min each, an hour of darkness was cumulatively delivered to the animal and this may have played a crucial role in the increase in REMS amount. However, an apparently compensatory decrease in REMS amount was observed in the following 18-h period, in which the proportion between light (6 h) and dark (12 h) was kept constant in both $\mathrm{N}$ and NDP conditions. This negative rebound should keep the 24-h REMS amount at comparable levels in the two conditions. The occurrence of a negative rebound confirms the presence of a strong homeostatic control of REMS occurrence on a relatively short-term basis (Amici et al., 1994, 1998; Cerri et al., 2005; Franken et al., 2002; Ocampo-Garcés et al., 2002).

The effect of ambient light intensity on REMS has also been observed under tonic experimental protocols in which darkness was tonically delivered. For example, the amount of REMS has been shown to increase under a condition of constant darkness, when the light is kept off during the normal 12-h light period, leading to a dark period which lasts for the whole day (Fishman and Roffwarg, 1972; Zamboni et al., 2001). In these experiments, no apparent compensatory reduction in the amount of REMS was observed in the following 24 h-period, when the animal was returned under a 
standard $12 \mathrm{~h}: 12 \mathrm{~h}$ LD cycle. However, the effect of the DP delivery protocol is difficult to compare with that of the continuous darkness protocol, due to the obvious absence of any rhythmicity in dark delivery and to a very large increase in the duration of darkness in the latter.

The dynamics of the REMS rebound during the 18-h recovery period at normal laboratory Ta that followed the $6 \mathrm{~h}$ period of exposure confirms those observed in previous experiments in this species (Amici et al., 1994, 1998; Cerri et al., 2005; Franken et al., 1993). Also, the REMS rebound following REMS deprivation was not apparently modified by the previous DP delivery. This suggests that the autonomic challenge caused by exposure to low Ta may be considered as a predominant factor with respect to the changes in ambient light condition in the determination of the REMS homeostatic drive.

NREMS occurrence was also modified during exposure to the random DP administration protocol. In fact, during the 6-h delivery period at $\mathrm{Ta} 25^{\circ} \mathrm{C}$, Delta Power levels were higher with respect to the unstimulated condition and the effect was statistically significant from 11 to $13 \mathrm{~h}$. Delta power levels during NREMS are considered to be an index of NREMS intensity and have been shown to be quantitatively related to the duration of the previous wakefulness (Borbély and Achermann, 2005). In the present study, only a $4 \%$ increase in the amount of Wake (or, reciprocally, a decrease in NREMS) was observed during the 6-h period of DP delivery compared to during the unstimulated condition. However, the size of this increase cannot reasonably account for the enhancement in Delta power during sNREMS, which occurred during the same 6-h period. On the other hand, although REMS occurrence was slightly enhanced during DP delivery, the extent to which REMS participates in the build up of the Delta power need in NREMS is still under discussion (Borbély and Achermann, 2005). A role for the 'quality' of Wake as a critical factor in the build up of Delta power need has been proposed. In fact, Delta Power has been shown to increase when the time spent in the previous period of waking was particularly stressful (Meerlo et al., 1997) or when it was characterized by an intense exploratory behaviour (Huber et al., 2007). While we can exclude that the time spent in wake during the 6-h period of DP delivery at Ta $25^{\circ} \mathrm{C}$ was stressful, due to the unimpaired occurrence of REMS, a possible explanation for the increase in Delta power may be linked to the fact that the aforementioned increase in exploratory behaviour reported in Huber et al. paper (2007) was achieved through exposure to a continuous darkness protocol. Thus, a possible increase in the exploratory behaviour induced by the rhythmical DP delivery and the extra-hour of darkness this entails would account for the increase in Delta power.

\section{Conclusions}

In conclusion, the results of this experiment confirm the effectiveness of the DP in inducing a REMS onset with a high probability and a short latency. Moreover, the similarity of the pattern of EEG and autonomic variables during the NREMS to REMS transition induced by a DP to that occurring spontaneously reinforces the hypothesis that such an effectiveness may be due to a direct influence of the DP on the neuronal network physiologically involved in spontaneous REMS generation (Rechtschaffen et al., 1969). Thus, the incapacity of DP to overcome the tonic depressing influence on REMS occurrence by central thermoregulatory activation underlines the crucial physiological meaning of the mutual exclusion of thermoregulatory activation and REMS occurrence, and supports the hypothesis that the suspension of the central control of body temperature is a prerequisite for REMS occurrence (Parmeggiani, 2005).

\section{ACKNOWLEDGEMENTS}

This work has been supported by grants from both Ministero dell'Università e della Ricerca Scientifica, Italy and the University of Bologna. The authors would like to thank $\mathrm{Mr}$ G. Mancinelli and Mr L. Sabattini for the wiring and the mechanical work needed for the adaptation of both the recording apparatus and the room.

\section{REFERENCES}

Alam, M. N., McGinty, D. and Szymusiak, R. Preoptic/anterior hypothalamic neurons: thermosensitivity in rapid eye movement sleep. Am. J. Physiol., 1995, 269: R1250-R1257.

Amici, R., Zamboni, G., Perez, E., Jones, C. A., Toni, I., Culin, F. and Parmeggiani, P. L. Pattern of desynchronized sleep during deprivation and recovery induced in the rat by changes in ambient temperature. J. Sleep Res., 1994, 3: 250-256.

Amici, R., Zamboni, G., Perez, E., Jones, C. A. and Parmeggiani, P. L. The influence of a heavy thermal load on REM sleep in the rat. Brain Res., 1998, 781: 252-256.

Amici, R., Jones, C. A., Perez, E. and Zamboni, G. A physiologic view of REM sleep structure. In: P. L. Parmeggiani and R. Velluti (Eds) The Physiologic Nature of Sleep. Imperial College Press, London, 2005: 161-185.

Baracchi, F., Amici, R., Capitani, P., Cerri, M., Dentico, D., Jones, C., Luppi, M., Martelli, D., Parmeggiani, P. L., Perez, E. and Zamboni, G. Cold exposure depresses dark pulse efficiency in triggering REM sleep in the albino rat. J. Sleep Res., 2004, 13(Suppl.1): 49.

Benca, R. M., Bergmann, B. M., Leung, C., Nummy, D. and Rechtschaffen, A. Rat strain differences in response to dark pulse triggering of paradoxical sleep. Physiol. Behav., 1990, 49: 83-87.

Benca, R. M., Gilliland, M. A. and Obermeyer, W. H. Effects of lighting conditions on sleep and wakefulness in albino Lewis and pigmented Brown Norway rats. Sleep, 1998, 21: 451-460.

Benington, J. H., Kodali, S. K. and Heller, H. C. Scoring transitions to REM sleep in rats based on the EEG phenomena of pre-REM sleep: an improved analysis of sleep structure. Sleep, 1994, 17: 28-36.

Borbély, A. A. Effects of light on sleep and activity rhythms. Prog. Neurobiol., 1978, 10: 1-31.

Borbély, A. A. and Achermann, P. Sleep homeostasis and models of sleep regulation. In: M. H. Kryger, T. Roth and W. E. Dement (Eds) Principles and Practice of Sleep Medicine. WB Saunders, Philadelphia, 2005: 405-417. 
Brown, R. E. and Mc Carley, R. W. Neurotransmitters, neuromodulators, and sleep. In: P. L. Parmeggiani and R. Velluti (Eds). The Physiologic Nature of Sleep. Imperial College Press, London, 2005: 45-75.

Capitani, P., Cerri, M., Amici, R., Baracchi, F., Jones, C. A., Luppi, M., Perez, E., Parmeggiani, P. L. and Zamboni, G. Changes in EEG activity and hypothalamic temperature as indices for nonREM sleep to REM sleep transitions. Neurosci. Lett., 2005, 383: 182-187.

Cerri, M., Ocampo-Garces, A., Amici, R., Baracchi, F., Capitani, P., Jones, C. A., Luppi, M., Perez, E., Parmeggiani, P. L. and Zamboni, G. Cold exposure and sleep in the rat: effects on sleep architecture and the electroencephalogram. Sleep, 2005, 28: 694-705.

Cornwall, J., Cooper, J. D. and Phillipson, O. T. Afferent and efferent connections of the laterodorsal tegmental nucleus in the rat. Brain Res. Bull., 1990, 25: 271-284.

Deboer, T., Ruijgrok, G. and Meijer, J. H. Short light-dark cycles affect sleep in mice. Eur. J. Neurosci., 2007, 26: 3518-3523.

Fishman, R. and Roffwarg, H. P. REM sleep inhibition by light in the albino rat. Exp. Neurol., 1972, 36: 166-178.

Fite, K. V., Janusonis, S., Foote, W. and Bengston, L. Retinal afferents to the dorsal raphe nucleus in rats and Mongolian gerbils. J. Comp. Neurol., 1999, 414: 469-484.

Fleming, M. D., Benca, R. M. and Behan, M. Retinal projections to the subcortical visual system in congenic albino and pigmented rats. Neuroscience, 2006, 143: 895-904.

Franken, P. Long-term vs. short-term processes regulating REM sleep. J. Sleep. Res., 2002, 11: 17-28.

Franken, P., Tobler, I. and Borbély, A. A. Effects of 12-h sleep deprivation and of 12-h cold exposure on sleep regulation and cortical temperature in the rat. Physiol. Behav., 1993, 54: 885-894.

Gottesmann, C. The transition from slow-wave sleep to paradoxical sleep: evolving facts and concepts of the neurophysiological processes underlying the intermediate stage of sleep. Neurosci. Biobehav. Rev., 1996, 20: 367-387.

Gvilia, I., Turner, A., Mcginty, D. and Szymusiak, R. Preoptic area neurons and the homeostatic regulation of rapid eye movement sleep. J. Neurosci., 2006, 26: 3037-3044.

Heller, H. C. Temperature, thermoregulation, and sleep. In: M. H. Kryger, T. Roth and W. E. Dement (Eds) Principles and Practice of Sleep Medicine. WB Saunders, Philadelphia, 2005: 292-304.

Huber, R., Tononi, G. and Cirelli, C. Exploratory behavior, cortical BDNF expression, and sleep homeostasis. Sleep, 2007, 30: 129139.

Ida, T., Nakahara, K., Murakami, T., Hanada, R., Nakazato, M. and Murakami, N. Possible involvement of orexin in the stress reaction in rats. Biochem. Biophys. Res. Commun., 2000, 270: 318323.

Johnson, J. H., Adler, N. T. and Sawyer, C. H. Effects of various photoperiods on the temporal distribution of paradoxical sleep in rats. Exp. Neurol., 1970, 27: 162-171.

Levine, J. D., Weiss, M. L., Rosenwasser, A. M. and Miselis, R. R. Retinohypothalamic tract in the female albino rat: a study using horseradish peroxidase conjugated to cholera toxin. J. Comp. Neurol., 1991, 306: 344-360.

Lisk, R. D. and Sawyer, C. H. Induction of paradoxical sleep by lightsoff stimulation. Proc. Soc. Exp. Biol. Med., 1966, 123: 664-667.

Lu, J., Greco, M. A., Shiromani, P. and Saper, C. B. Effect of lesions of the ventrolateral preoptic nucleus on NREM and REM sleep. $J$. Neurosci., 2000, 20: 3830-3842.

Lu, J., Bjorkum, A. A., Xu, M., Gaus, S. E., Shiromani, P. J. and Saper, C. B. Selective activation of the extended ventrolateral preoptic nucleus during rapid eye movement sleep. J. Neurosci., 2002, 22: 4568-4576.

Lu, J. W., Fenik, V. B., Branconi, J. L., Mann, G. L., Rukhadze, I. and Kubin, L. Disinhibition of perifornical hypothalamic neurones activates noradrenergic neurones and blocks pontine carbachol- induced REM sleep-like episodes in rats. J. Physiol., 2007, 582: 553567.

Lund, R. D. Uncrossed visual pathways of hooded and albino rats. Science, 1965, 149: 1506-1507.

McCarley, R. W. and Hobson, J. A. Cortical unit activity in desynchronized sleep. Science, 1970, 167: 901-903.

Meerlo, P., Pragt, B. J. and Daan, S. Social stress induces high intensity sleep in rats. Neurosci. Lett., 1997, 225: 41-44.

Miller, A. M., Chappell, R., Obermeyer, W. H. and Benca, R. M. Analysis of the retinohypothalamic tract in congenic albino and pigmented rats. Brain Res., 1996, 741: 348-351.

Miller, A. M., Obermeyer, W. H., Behan, M. and Benca, R. M. The superior colliculus-pretectum mediates the direct effects of light on sleep. Proc. Natl Acad. Sci. U. S. A., 1998, 95: 8957-8962.

Miller, A. M., Miller, R. B., Obermeyer, W. H., Behan, M. and Benca, R. M. The pretectum mediates rapid eye movement sleep regulation by light. Behav. Neurosci., 1999, 113: 755-765.

Morin, L. P. and Allen, C. N. The circadian visual system, 2005. Brain Res. Rev., 2006, 51: 1-60.

Ocampo-Garces, A. and Vivaldi, E. A. Short-term homeostasis of REM sleep assessed in an intermittent REM sleep deprivation protocol in the rat. J. Sleep Res., 2002, 11: 81-89.

Parmeggiani, P. L. Interaction between sleep and thermoregulation: an aspect of the control of behavioral states. Sleep, 1987, 10: 426.

Parmeggiani, P. L. Thermoregulation and sleep. Front. Biosci., 2003, 8: s557-s567.

Parmeggiani, P. L. Physiologic regulation in sleep. In: M. H. Kryger, T. Roth and W. E. Dement (Eds) Principles and practice of sleep medicine. WB Saunders, Philadelphia, 2005: 185-191.

Parmeggiani, P. L. REM sleep related increase in brain temperature: a physiologic problem. Arch. Ital. Biol., 2007, 145: 13-21.

Parmeggiani, P. L., Agnati, L. F., Zamboni, G. and Cianci, T. Hypothalamic temperature during the sleep cycle at different ambient temperatures. Electroencephalogr. Clin. Neurophysiol., 1975, 38: 589-596.

Perez, E., Zamboni, G., Amici, R., Fadiga, L. and Parmeggiani, P. L. Ultradian and circadian changes in the cAMP concentration in the preoptic region of the rat. Brain Res., 1991, 551: 132-135.

Prichard, J. R., Fahy, J. L., Obermeyer, W. H., Behan, M. and Benca, R. M. Sleep responses to light and dark are shaped by early experience. Behav. Neurosci., 2004, 118: 1262-1273.

Prichard, J. R., Armacanqui, H. S., Benca, R. M. and Behan, M. Light-dependent retinal innervation of the rat superior colliculus. Anat. Rec. (Hoboken), 2007, 290: 341-348.

Rechtschaffen, A., Dates, R., Tobias, M. and Whitehead, I. The effects of lights-off stimulation on the distribution of paradoxical sleep in the rat. Communs. Behav. Biol., 1969, 3: 93-99.

Simerly, R. B. and Swanson, L. W. Projections of the medial preoptic nucleus: a Phaseolus vulgaris leucoagglutinin anterograde tracttracing study in the rat. J. Comp. Neurol., 1988, 270: 209-242.

Sisk, C. L. and Stephan, F. K. Central visual pathways and the distribution of sleep in 24-hr and 1-hr light-dark cycles. Physiol. Behav., 1982, 29: 231-239.

Steriade, M. and Mc Carley, R. W. Brainstem Control of Wakefulness and Sleep. Plenum Press, New York, 1990.

Szymusiak, R., Gvilia, I. and McGinty, D. Hypothalamic control of sleep. Sleep Med, 2007, 8: 291-301.

Terenzi, M. G., Zagon, A. and Roberts, M. H. Efferent connections from the anterior pretectal nucleus to the diencephalon and mesencephalon in the rat. Brain Res., 1995, 701: 183-191.

Trachsel, L., Tobler, I. and Borbély, A. A. Electroencephalogram analysis of non-rapid eye movement sleep in rats. Am. J. Physiol., 1988, 255: R27-R37.

Wallenstein, S., Zucker, C. L. and Fleiss, J. L. Some statistical methods useful in circulation research. Circ. Res., 1980, 47: 1-9.

Wang, Q. P., Guan, J. L. and Shioda, S. Synaptic contacts between serotonergic and cholinergic neurons in the rat dorsal raphe nucleus 
and laterodorsal tegmental nucleus. Neuroscience, 2000, 97: 553563.

Yoshida, K., Mccormack, S., Espana, R. A., Crocker, A. and Scammell, T. E. Afferents to the orexin neurons of the rat brain. J. Comp. Neurol., 2006, 494: 845-861.

Zamboni, G., Amici, R., Perez, E., Jones, C. A. and Parmeggiani, P. L. Pattern of REM sleep occurrence in continuous darkness following the exposure to low ambient temperature in the rat. Behav. Brain Res., 2001, 122: 25-32.

Zamboni, G., Jones, C. A., Domeniconi, R., Amici, R., Perez, E., Luppi, M., Cerri, M. and Parmeggiani, P. L.. Specific changes in cerebral second messenger accumulation underline REM sleep inhibition induced by the exposure to low ambient temperature. Brain Res., 2004, 1022: 62-70. 